Open Access

\title{
Radiotherapy for asymptomatic brain metastasis in epidermal growth factor receptor mutant non-small cell lung cancer without prior tyrosine kinase inhibitors treatment: a retrospective clinical study
}

SongRan Liu ${ }^{1,2,3+}$, Bo Qiu ${ }^{1,2,3+}$, LiKun Chen ${ }^{1,2,4}$, Fang Wang ${ }^{1,2,5}$, Ying Liang ${ }^{1,2,4}$, PeiQiang Cai ${ }^{1,2,6}$, Li Zhang ${ }^{1,2,4}$,

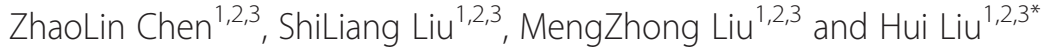

\begin{abstract}
Background: Non-small cell lung cancer (NSCLC) with brain metastasis (BM) harboring an epidermal growth factor receptor (EGFR) mutation shows good response to tyrosine kinase inhibitors (TKIs). This study is to assess the appropriate timing of brain radiotherapy (RT) for asymptomatic BM in EGFR mutant NSCLC patients.

Methods: There were 628 patients diagnosed with EGFR mutant NSCLC between October 2005 and December 2011. Treatment outcomes had been retrospectively evaluated in 96 patients with asymptomatic BM without prior TKI treatment. 39 patients received first-line brain RT, 23 patients received delayed brain RT, and 34 patients did not receive brain RT.

Results: With a median follow-up of 26 months, the 2-year OS was $40.6 \%$. Univariate analyses revealed that ECOG performance status $(p=0.006)$, other distant metastases $(p=0.002)$ and first line systemic treatment $(p=0.032)$ were significantly associated with overall survival (OS). Multivariate analyses revealed that other sites of distant metastases $(p=0.030$ ) were prognostic factor. The timing of brain RT was not significantly related to OS $(p=0.246)$. The 2-year BM progression-free survival (PFS) was $26.9 \%$. Brain RT as first-line therapy failed to demonstrate a significant association with BM PFS ( $p=0.643)$.
\end{abstract}

Conclusions: First-line brain RT failed to improve long-term survival in TKI-naïve EGFR mutant NSCLC patients with asymptomatic BM. Prospective studies are needed to validate these clinical findings.

Keywords: Asymptomatic brain metastasis, Radiotherapy, Chemotherapy, Epidermal growth factor receptor mutation, Tyrosine kinase inhibitor

\footnotetext{
* Correspondence: liuhui@sysucc.org.cn

${ }^{\dagger}$ Equal contributors

${ }^{1}$ State Key Laboratory of Oncology in South China, Collaborative Innovation Center for Cancer Medicine, Guangzhou 510060, China

${ }^{2}$ Guangdong Esophogeal Cancer Research Institute, Guangzhou, China

Full list of author information is available at the end of the article
} 


\section{Background}

Brain metastasis (BM) is a common complication of lung cancer and is associated with poor treatment outcomes. $\mathrm{BM}$ is observed in approximately $25-30 \%$ of non-small cell lung cancer (NSCLC) patients [1]. The median survival is approximately $4-11$ weeks in untreated patients but can be improved by whole-brain radiation therapy (WBRT) to 3-6 months [2]. However, NSCLC has been regarded as a relatively radio-resistant malignancy, and 30 Gy WBRT may be insufficient to destroy the lesions; recent studies have suggested that the median response rate to WBRT remains approximately $25-30 \%$ [3]. The role of chemotherapy in the treatment of brain metastasis remains controversial.

Advances in the understanding of the molecular biology of tumors have led to the development of targeted agents with promising results in the treatment of NSCLC. Epidermal growth factor receptor (EGFR) mutations are associated with a significant sensitivity to EGFR tyrosine kinase inhibitors (TKI), which can significantly improve treatment outcome [4]. Recently, the efficacy of epidermal growth factor receptor tyrosine kinase inhibitors (EGFR-TKIs) for NSCLC patients with BM has been reported [5, 6]. Moreover, several reports demonstrate that NSCLC patients with mutant EGFR and BM could also achieve favorable outcomes when treated with EGFR-TKIs as single-agent chemotherapy. Several studies have reported that TKI treatment results in high response rates (70-89\%) and increased overall survival (OS) and progression-free survival (PFS) (12.9-19.8 months and 6.6-23.3 months, respectively) in selected populations of EGFR-mutated NSCLC patients with BM [7-9].

Several studies have suggested that patients with BM harboring EGFR mutations may have higher response rates to WBRT than those with wild-type tumors [10-13]. However, unlike the EGFR-mutant primary lung tumor, $11-44 \%$ of brain metastases exhibit resistance to TKI treatment $[7,8]$. In addition, Omuro et al. [14] reported a high incidence of central nervous system (CNS) metastases during the course of a standard treatment of gefitinib, an EGFR inhibitor, despite good control of other disease sites. These results suggest that local therapy may still be important for the treatment of BM in patients with EGFR mutations.

However, for EGFR-mutant NSCLC patients with asymptomatic brain metastasis who do not require urgent symptom relief, the proper treatment schedule is not well established. Therefore, we sought to gain insight from the retrospective analysis of patients treated with different combinations of irradiation/TKI therapies.

\section{Methods}

Acquisition of clinical data

A total of 628 patients were diagnosed with adenocarcinoma of the lung harboring EGFR mutations between
October 2005 and December 2011 at the Sun Yat-Sen University Cancer Center. Treatment outcomes had been retrospectively evaluated in 96 patients with asymptomatic BM without prior TKI treatment. Before receiving treatment, each patient underwent a physical examination, laboratory tests and electrocardiograms as well as a medical history evaluation, including documentation of concomitant medications, performance status, and smoking history. Patient data included chest and upper abdomen computed tomography (CT) scans or positron emission tomography (PET) scans, bone scans, and magnetic resonance imaging (MRI) of the brain. Tumor stage was classified using the tumor/node/metastasis (TNM) system proposed by the American Joint Committee on Cancer (8th edition). $\mathrm{T}$ and $\mathrm{N}$ stage were determined on the basis of the findings of CT with or without additional fiberoptic bronchoscopy. Mediastinal lymph nodes $\geq 1 \mathrm{~cm}$ on transaxial CT images or SUV $\geq$ 2.5 on PET scans were considered positive. All patients were required to meet the following inclusion criteria: 1 ) pathologically confirmed NSCLC harboring an activating EGFR mutation; 2) documented measurable brain metastases (AJCC stage IV disease) at first diagnosis; and 3) Eastern Cooperative Oncology Group (ECOG) performance status $\leq 3$. Patients with severe comorbid conditions and other active malignancies were excluded from the analysis.

\section{EGFR Genotyping}

All patients provided written informed consent for the comprehensive use of molecular analysis. Genomic DNA was isolated from paraffin-embedded tissues, which were obtained by transbronchial lung biopsy or from cytologic materials, such as bronchial lavage fluid and pleural effusions. Methanol-fixed cytologic specimens were used for DNA extraction for patients for whom only cytologic sample were available at initial diagnosis. Epidermal growth factor receptor mutation analysis of all of the patients was performed by direct sequencing or the ARMS method at the Department of Molecular Diagnosis [15].

\section{Follow-up and treatment response assessment}

The beginning of the follow-up period was defined as the initial date of local or systemic treatment. Patients underwent chest, abdomen, and pelvic $\mathrm{CT}$ and brain MRI every three months until disease progression. Bone scans were administered when patients were suspected of having bone metastasis. Positron emission tomography scans were administered when systemic progression was expected. The rates and times of treatment response, overall survival, local relapse and distant metastases were recorded.

Systemic disease at the time of BM diagnosis was considered active if a chest, abdomen and pelvic CT, PET, 
and/or bone scan performed within four weeks of the $\mathrm{BM}$ diagnosis revealed new sites of extra-cranial metastases or progression at previously known sites of disease. The time to brain progression was measured from the initial date of local or systemic treatment until the date of radiological progression or worsening neurological symptoms at the time of last follow-up.

\section{Statistical analysis}

The study endpoint was OS. Overall survival was calculated as the time from the initial date of local or systemic treatment to the date of death from any cause or to the last visit before March 31, 2014, censored at the date of last follow up. Each variable was assessed first in univariate analysis, and the variables with a $\mathrm{P}$ value $<0.10$ were evaluated by multivariate analysis. Survival curves were plotted using the Kaplan-Meier method. We fit the proportional hazards model using Cox regression. After testing for variable interactions, a forward stepwise elimination procedure was used to determine the best-fitting model. $P$ values $<0.05$ were regarded as statistically significant in multivariate analysis. All statistical analyses were performed using SPSS 19.0 software (IBM).

\section{Ethics statement}

Participants' information collection was guaranteed by Ethics Committee of Sun Yat-sen University Cancer Center. Written informed consent was not obtained, instead, all clinical records was anonymized and de-identified prior to analysis. All authors have read and approved the manuscript.

\section{Results}

\section{Patient characteristics}

The patient characteristics are detailed in Table 1 . The study included 96 patients, with 62 females and 34 males. There were 50 patients with $\mathrm{BM}$ at the initial diagnosis of NSCLC (stage IV), the other 46 patients developed $\mathrm{BM}$ after first-line treatment. None of the patients received TKI therapy before the diagnosis of BM. None of the patients experienced any symptoms related to the metastatic brain tumors. After diagnosis and general evaluation, all 96 patients received either chemotherapy or a TKI as systemic treatment. The regimens included cisplatin or carboplatin combined with paclitaxel or pemetrexed. All 61 patients exhibited disease progression and were administered a TKI as second-line systemic treatment after the diagnosis of BM. The 35 patients who received TKI as first-line therapy after the diagnosis of BM were treated with $250 \mathrm{mg}$ of oral gefitinib or $150 \mathrm{mg}$ of erlotinib once daily until disease progression or unacceptable toxicity.
Table 1 Clinical and molecular characteristics of patients $(n=96)$

\begin{tabular}{|c|c|}
\hline Charateristics & Patients $(n=96)$ No. $(\%)$ \\
\hline \multicolumn{2}{|l|}{ Sex } \\
\hline Male & $34(35.4 \%)$ \\
\hline Female & $62(64.6 \%)$ \\
\hline Age(year), median(range) & $54(26-79)$ \\
\hline \multicolumn{2}{|l|}{${ }^{\mathrm{a} E C O G}$ performance status } \\
\hline $0-1$ & $69(71.9 \%)$ \\
\hline $2-3$ & $27(28.1 \%)$ \\
\hline \multicolumn{2}{|l|}{ No. of brain lesions } \\
\hline $1-4$ & $32(33.3 \%)$ \\
\hline$>4$ & $64(66.7 \%)$ \\
\hline \multicolumn{2}{|l|}{ Max size of brain lesions } \\
\hline$<1 \mathrm{~cm}$ & 75 (78.1 \%) \\
\hline$>1 \mathrm{~cm}$ & $21(21.9 \%)$ \\
\hline \multicolumn{2}{|l|}{ Location of brain lesions } \\
\hline Brain stem mets & $13(13.5 \%)$ \\
\hline Non-brain stem mets & $83(86.5 \%)$ \\
\hline \multicolumn{2}{|c|}{ Other sites of metastatic disease } \\
\hline Yes & $61(63.5 \%)$ \\
\hline No & $35(36.5 \%)$ \\
\hline \multicolumn{2}{|l|}{ bEGFR mutation } \\
\hline Exon 19 & $45(46.9 \%)$ \\
\hline Exon 21 & $51(53.1 \%)$ \\
\hline \multicolumn{2}{|l|}{ First line systemic treatment } \\
\hline Chemotherapy & $61(63.5 \%)$ \\
\hline${ }^{\mathrm{C}} \mathrm{TKI}$ & $35(36.5 \%)$ \\
\hline \multicolumn{2}{|l|}{ Time of Brain radiotherapy } \\
\hline First line & $39(40.6 \%)$ \\
\hline Delayed & $23(24.0 \%)$ \\
\hline $\mathrm{No}{ }^{\mathrm{d}} \mathrm{RT}$ & $34(35.4 \%)$ \\
\hline
\end{tabular}

aEastern Cooperative Oncology Group

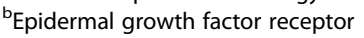

'Tyrosine kinase inhibitors

Radiotherapy

\section{Survival and prognostic factors}

With a median follow-up of 26 months (range, 11-56 months), the 2-year OS was $40.6 \%$, and the estimated OS time was 21.0 months (Fig. 1). Eastern Cooperative Oncology Group performance status $(p=0.006)$, other distant metastases $(p=0.002)$ and first line systemic treatment $(\mathrm{p}=0.032)$ were significantly associated with OS (Table 2). The clinical factors that were statistically significant $(\mathrm{p}<0.10)$ in a univariate analysis were analyzed further in a multivariate analysis with a stepwise selection of variables. Only other sites of distant metastases $(p=0.030)$ were selected by a stepwise selection of factors in the final models (Table 3). 


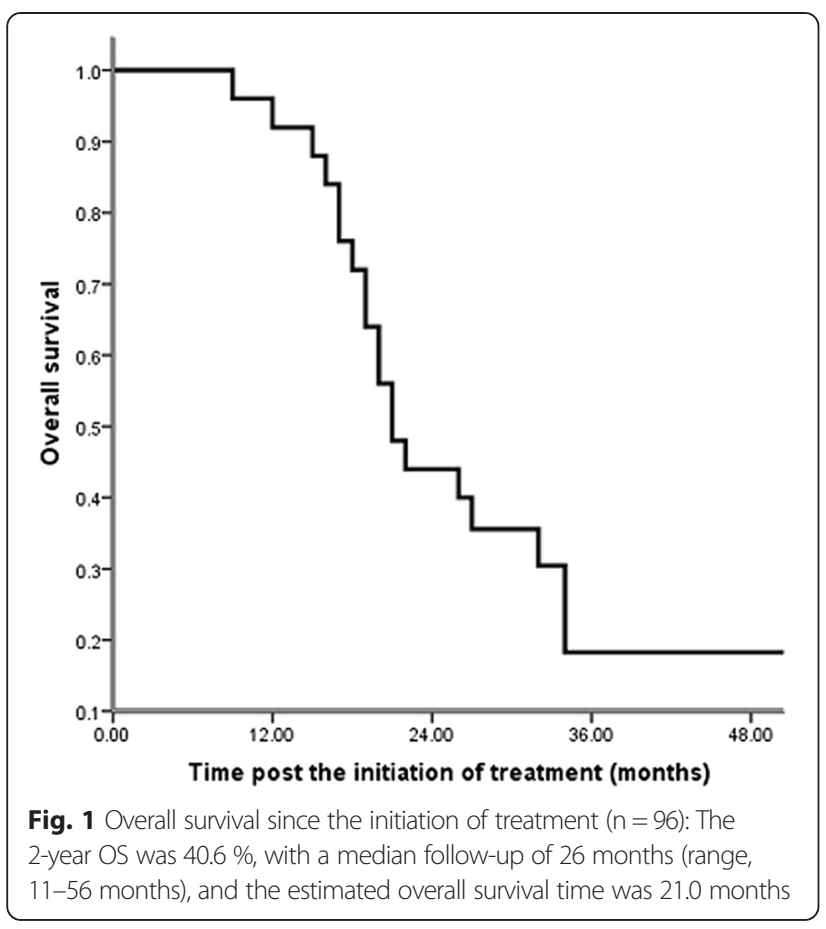

Table 2 Univariate analysis of prognostic factors of overall survival $(n=96)$

\begin{tabular}{lcc}
\hline Variable & HR, $95 \% \mathrm{Cl}$ & $\mathrm{p}$ value \\
\hline Sex & $0.81(0.43-1.55)$ & 0.534
\end{tabular}

(male vs. female)

Age

$0.74(0.39-1.41) \quad 0.342$

( $>54$ years vs. $\leq 54$ years)

ECOG performance status

$3.24(1.40-7.50) \quad 0.006$

(0-1 vs. 2-3)

No. of brain metastases

$1.41(0.74-2.69) \quad 0.299$

$(1-4$ vs. $>4)$

Max size of brain lesions

$1.03(0.67-1.76) \quad 0.763$

$(<1 \mathrm{~cm}$ vs. $>1 \mathrm{~cm})$

Location of brain lesions

$1.62(0.98-2.73) \quad 0.267$

(Brain stem mets vs. Non-brain stem mets)

Other sites of metastatic disease

$3.89(1.58-9.57) \quad 0.002$

(No vs. Yes)

EGFR mutation

$0.74(0.39-1.40) \quad 0.351$

(Exon 19 vs. Exon 21)

First line systemic treatment

$2.43(1.08-5.48) \quad 0.032$

(TKI vs. Chemotherapy)

Time of brain RT

$0.77(0.50-1.19)$

0.246

(First line vs. Delayed vs. No RT)

Legend: Each variable was assessed first in univariate analysis, with $\mathrm{P}$ value $<0.10$ regarded as statistically significant. Instead of timing of brain radiotherapy (RT), only Eastern Cooperative Oncology Group (ECOG) performance status, other distant metastases and first line systemic treatment were significantly associated with the overall survival
Table 3 Multivariate analysis of prognostic factors for overall survival $(n=96)$

\begin{tabular}{llc}
\hline Variable & $\mathrm{HR}, 95 \% \mathrm{Cl}$ & $\mathrm{p}$ value \\
\hline Other distant metastases & $3.53(1.13-11.08)$ & 0.030 \\
(No vs. Yes) & &
\end{tabular}

Legend: The clinical factors that were statistically significant $(p<0.10)$ in a univariate analysis were analyzed further in a multivariate analysis with a stepwise selection of variables. $P$ values $<0.05$ were regarded as statistically significant in multivariate analysis. Only other sites of distant metastases were selected by a stepwise selection of factors in the final models

\section{Difference in outcomes by brain RT}

Thirty-nine patients were treated initially with brain RT (13 with stereotactic radiosurgery and 26 with WBRT), and 23 patients were administered RT when they exhibited brain disease progression (eight with stereotactic radiosurgery and 15 with WBRT). Thirty-four patients did not receive radiotherapy and remained asymptomatic until the last follow-up. Univariate analysis revealed that the timing of brain RT was not significantly related to OS ( $p=0.246$, Table 2, Fig. 2). During the follow-up, 54 patients exhibited progressive brain disease after local or systemic treatment. The 2-year BM PFS was $29.6 \%$, and the estimated BM PFS time was 17 months (Fig. 3). Brain RT as first-line therapy was not significantly associated with $\mathrm{BM}$ progression-free survival $(\mathrm{p}=0.643, \mathrm{HR}$ 0.82, 95 \% CI 0.39-1.81, Fig. 4).

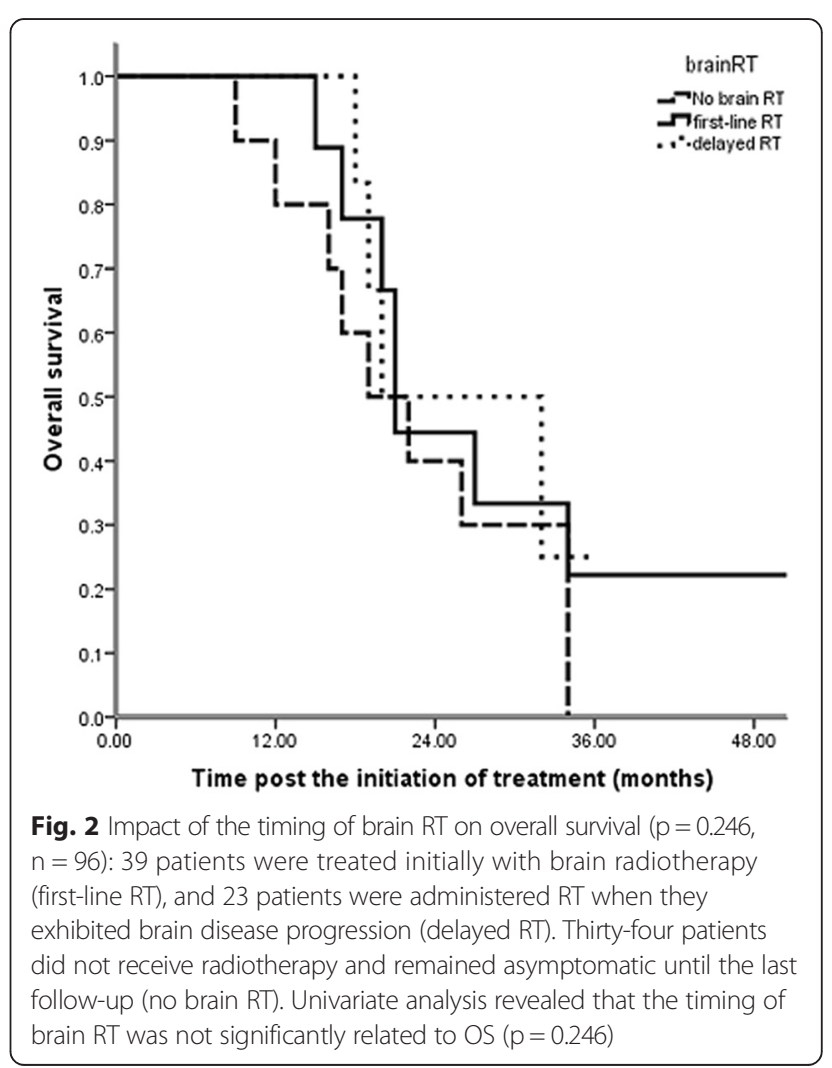




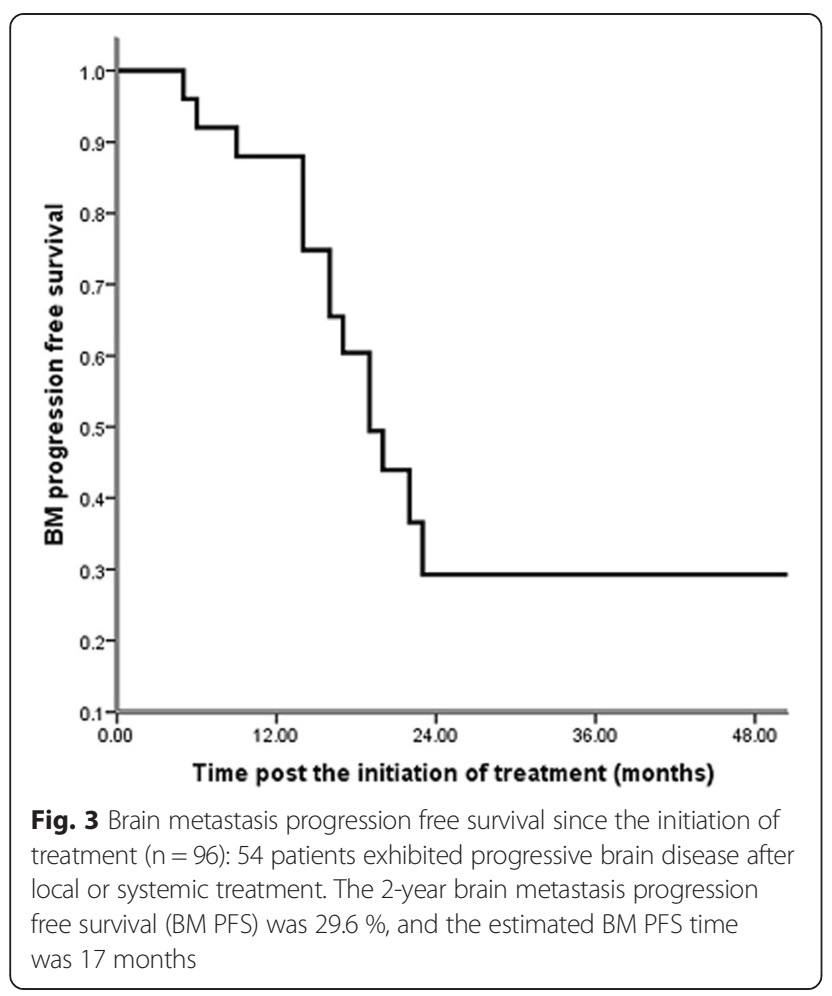

There were 35 patients with brain metastases only, 19 patients received immediate brain RT, eight patients had delayed brain RT, and eight patients did not receive brain RT. Univariate analysis showed that the timing of brain RT was significantly related to OS $(\mathrm{P}=0.019$,

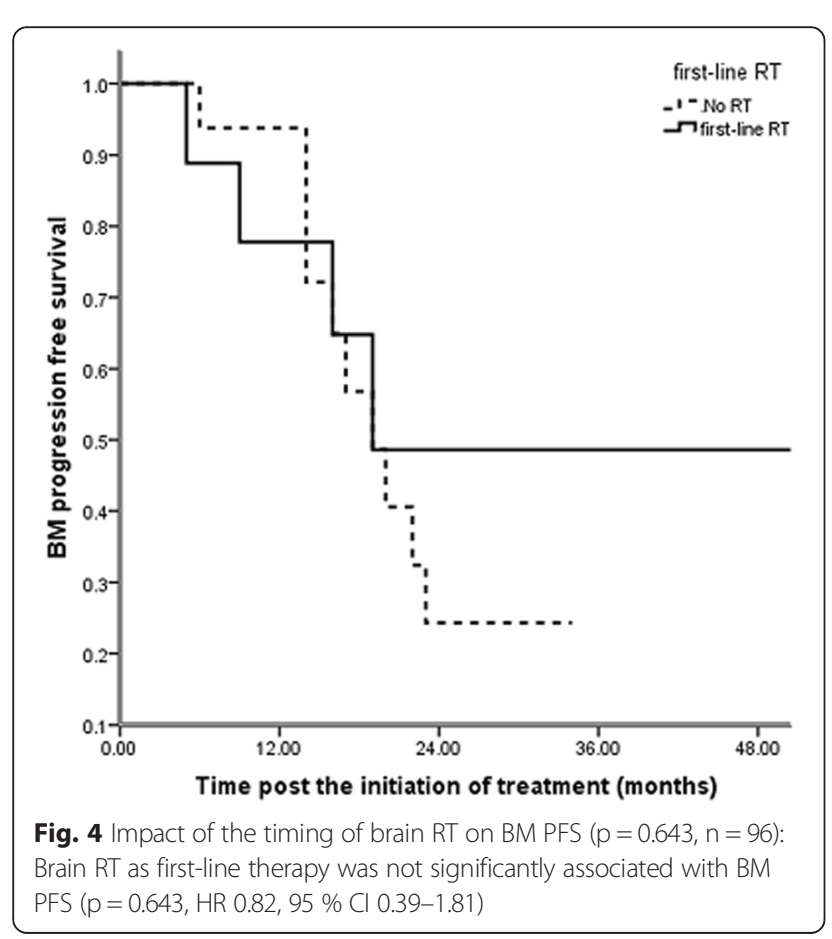

Fig. 5). Patients with delayed brain RT had better longterm survival than the others.

\section{Pattern of treatment failure}

Of the $72(72 / 96,75 \%)$ patients who died during followup, 65 (65/96, $68 \%)$ patients died of disease progression, and the remaining seven died of unknown reasons. Among the 65 patients, 46 (46/65, $71 \%)$ patients exhibited extra-cranial lesion progression, seven $(7 / 65,11 \%)$ patients exhibited intra-cranial lesion progression only, and $12(12 / 65,18 \%)$ patients exhibited both intra- and extra-cranial lesion progression. Patients received crossover therapies (TKI-Chemotherapy or ChemotherapyTKI) when they exhibited systemic disease progression.

\section{Toxicities}

The most frequent toxicities observed were nausea, vomiting, neutropenia and skin rash, and the majority of toxicities were Grade 2 (G2). Grade 3 vomiting was observed in 24 out of 96 patients ( $25 \%)$. Grade 3 neutropenia occurred in 23 out of 96 patients (24\%). Grade 3 skin rash was observed in 4 out of 96 patients (4.0\%). No G3-5 neurotoxicity was reported for patients who received brain RT. No treatment-related deaths were recorded.

\section{Discussion}

This study retrospectively analyzed a consecutive cohort of NSCLC patients tested for EGFR mutations. Our results suggest that among the EGFR-mutated patients

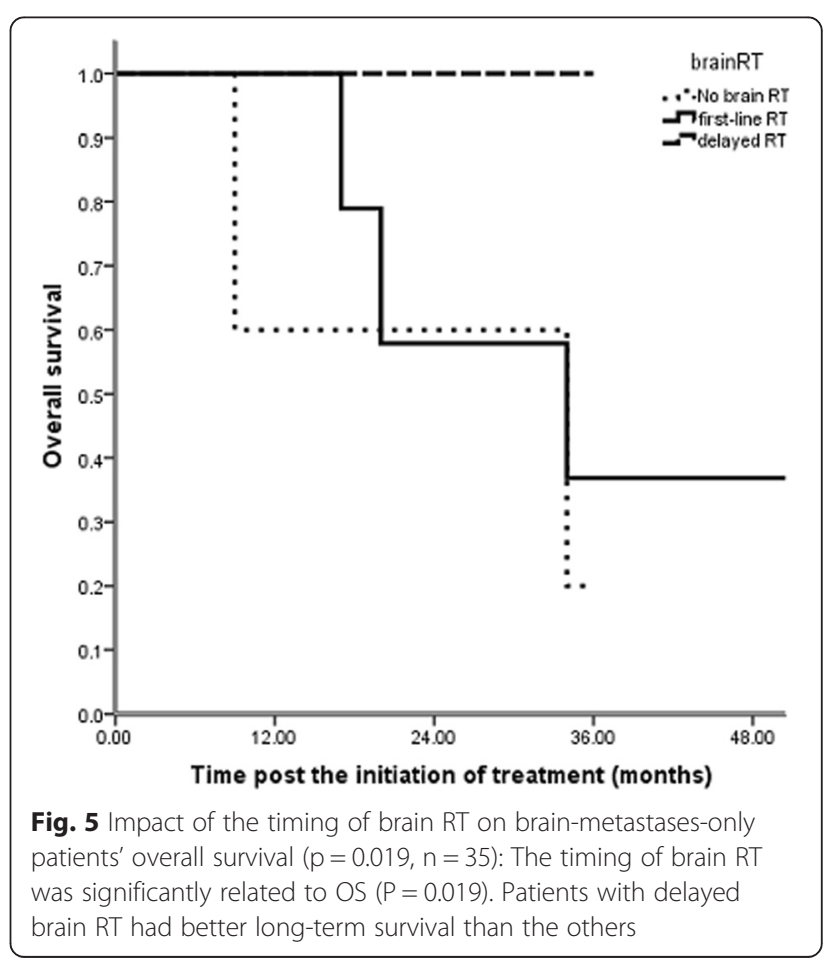


with asymptomatic BMs, the timing of brain RT was not significantly related to OS $(\mathrm{p}=0.246)$. Furthermore, subgroup analysis revealed that brain RT as first-line therapy was not significantly associated with $\mathrm{BM}$ progressionfree survival ( $p=0.643$ ). Only the status of other sites of distant metastases strongly influenced survival after the commencement of treatment $(\mathrm{p}=0.030)$.

Whole brain radiotherapy is generally the standard treatment in patients with multiple brain metastases, as it addresses both macroscopic and microscopic disease. Whole brain radiotherapy was shown to result in an improvement in symptoms in $64-85 \%$ of patients [16-18] and in a prolonged median survival of 3-6 months [2]. However, WBRT exhibits both short- and long-term toxicity. Patients may experience continued fatigue, nausea, neurocognitive deficits and other general or focal neurologic symptoms during and after the treatment $[19,20]$. Soffietti et al. reported the health-related quality-of-life (HRQOL) results of adjuvant WBRT compared with observation after either surgery or radiosurgery for a limited number of brain metastases in patients with stable solid tumors. Overall, patients in the observation arm reported better HRQOL scores than those who received WBRT. The differences were clinically relevant during the early follow-up period (for global health status at 9 months, physical functioning at 8 weeks, cognitive functioning at 12 months, and fatigue at 8 weeks) [21].

The prevalence of EGFR mutations in BM from NSCLC patients remains to be determined. Matsumoto et al. [22] and Gow et al. [11] have reported EGFR mutations in $63 \%$ and $44 \%$ of BM in an Asian study cohort, respectively. This prevalence is similar to that reported in primary lung lesions, varying from $30 \%$ to $50 \%$ [23, 24]. Eichler et al. [10] reported that in a cohort of 93 NSCLC patients with BM, 41 patients $(44 \%)$ had mutations in EGFR. The study demonstrated that a large portion of NSCLC patients with BMs exhibited EGFR mutations. Solitary BMs in the absence of other distant metastases were more common in NSCLC patients with wild-type EGFR compared with patients with mutated EGFR (31 vs. $7 \%$, and 35 vs. $12 \%$, respectively). In the study by Eichler et al., $18 \%$ of patients with BM were treated initially with WBRT either alone (53\%) or in combination with craniotomy for neurosurgical resection $(22 \%)$ or stereotactic radiosurgery $(8 \%)$. The median survival from the time of $\mathrm{BM}$ was 11.7 months and was longer for patients with an EGFR mutation (14.5 vs. 7.6 months, $\mathrm{p}=0.09)$. Multivariable analysis suggested that an EGFR mutation (HR: 0.50, 95 \% CI: 0.30-0.82), age (HR: 1.03, 95 \% CI: 1.00-1.05), and active extra-cranial disease (HR: 3.30, 95 \% CI: 1.706.41) were independently associated with survival. Gow et al. [11] retrospectively analyzed 63 NSCLC patients with BM treated by WBRT and found that 46 patients carrying EGFR mutations exhibited a median survival of
17.3 months compared with 6.6 months for patients with wild-type EGFR. Additionally, $54 \%$ of patients with EGFR mutations responded to WBRT compared with only $24 \%$ in the wild-type group.

Recent studies have reported that despite the issue of the blood brain barrier, patients with EGFR-mutated BM exhibit positive responses to TKIs in addition to the promising response rate to brain RT. Preclinical studies in mouse models of EGFR-mutant NSCLC BM have demonstrated the treatment efficacy of gefitinib. [25] A recent phase II study with 28 patients [26] prospectively evaluated the efficacy of EGFR TKIs in the treatment of metastatic brain tumors in NSCLC patients harboring EGFR mutations. Twenty-three patients $(83 \%)$ exhibited a partial response (PR), and 3 patients (11\%) had stable disease (SD), representing a disease control rate of $93 \%$. The median PFS and OS were 6.6 months $(95 \% \mathrm{CI}$, 3.8-9.3 months) and 15.9 months (95 \% CI, 7.224.6 months), respectively. There was no difference in PFS and OS with respect to the EGFR TKI used. After discontinuation of the treatment, 14 patients $(50 \%)$ received local therapy (whole-brain radiotherapy or radiosurgery) for BM during their disease course, with a local therapy-free interval of 12.6 months (95 \% CI, 7.617.6 months). The results implied that EGFR TKI therapy may represent the treatment of choice for BMs in NSCLC patients harboring an activating EGFR mutation. Gerber et al. also reported the results of erlotinib versus radiation therapy for brain metastases in 110 patients with EGFR-mutant lung adenocarcinoma and found that there was no significant difference in OS between the WBRT and erlotinib groups (median, 35 vs. 26 months; $\mathrm{P}=0.62)$ [27].

In the current study, 23 (23/96, 24 \%) patients received delayed brain RT, because the brain tumors were stable radiologically with no obvious neurologic symptoms during initial systemic treatment. Among these 23 patients, $13(13 / 23,56.5 \%)$ exhibited responses to brain RT, $6(6 / 23,26.1 \%)$ had stable disease, and $4(4 / 23$, $17.4 \%)$ developed intra-cranial disease progression. Four $(4 / 23,17.4 \%)$ patients died of intra-cranial disease progression, and $11(11 / 23,47.8 \%)$ patients died of active distant metastases in other sites. As not all patients received brain RT during the disease course and given the long-term neurotoxicity of WBRT and stereotactic radiosurgery, a better treatment option may be upfront treatment with EGFR TKIs followed by brain RT at the appropriate time when neurologic symptoms or signs develop. There were 35 patients with brain metastases only in current study, 19 patients received immediate brain RT, 8 patients had delayed brain RT, and 8 patients did not receive brain RT. Patients in this subgroup were more likely to have aggressive treatment. It might because patients with brain metastasis only were believed 
to have better treatment outcome than those with multiple sites metastases. However, univariate analysis showed that patients with delayed brain RT had better long term survival. This result suggested that sequential treatment (TKI followed by brain RT when brain lesions progress) might be an optimal choice for these patients. Since the number of patients in this subgroup remained limited, further studies should be conducted on this finding in the future.

Tumors with exon 19 deletions exhibited a higher incidence of CNS involvement compared with tumors bearing the L858R mutation (21 vs. $3 \%$ ). Sekine et al. [28] reported that compared with the wild-type EGFR group and the exon 21 point mutation group, NSCLC patients with the exon 19 deletion exhibit a peculiar pattern of brain metastases, including multiple small metastases with little brain edema. This metastatic pattern may be similar to that of miliary brain metastases. In our study, we included 45 patients with the exon 19 deletion and 51 patients with the exon 21 point mutation. Among the 64 patients with multiple (more than 4) BM lesions, 36 patients exhibited the exon 19 deletion, and 28 patients had the exon 21 point mutation. There was no significant difference between the two EGFR mutation types with respect to the numbers of BMs $(\mathrm{p}=0.934)$. Considering all 96 patients, we failed to observe any significant differences in OS ( $\mathrm{p}=0.362$, HR $0.75,95 \%$ CI 0.39-1.41) between the two types of EGFR mutations. The mechanism explaining the difference in intra-cranial spread remains unknown. Epidermal growth factor receptor mutations with the exon 19 deletion have been proposed to reduce the growth capacity of tumor cells, leading to smaller-sized BMs. In our study, we did not observe this phenomenon, possibly because only patients free of CNS symptoms were enrolled in the analysis.

There are several potential limitations of our current study. First, patients enrolled in the study received treatment between October 2005 and December 2011; therefore, 61 patients were administered chemotherapy as first-line systemic treatment, and only 35 patients received TKIs as first-line treatment. Most of the patients received crossover regimens (chemotherapy-TKI or TKIchemotherapy). Second, although recent studies reported a discordance rate of $27-28 \%$ for the EGFR mutation status between the primary and metastatic sites [29, 30], pathological and genetic confirmation of BM could not be performed. Third, there were several other limitations, including various chemotherapy regimens and the short follow-up time. Fourth, there may have been a selection bias with respect to patients requiring upfront RT and those patients in whom RT could be delayed. It is possible that the patients with deferred RT did well because they had a lower burden of CNS disease and did not require early WBRT.

\section{Conclusions}

In conclusion, this study revealed that EGFR TKIs are very effective for treating NSCLC patients harboring EGFR mutations and asymptomatic brain metastases. First-line brain RT did not improve long-term survival in this cohort of patients. The status of systemic disease was the strongest prognostic factor for stage IV EGFRmutated NSCLC patients. In TKI-naïve patients with an EGFR mutation and asymptomatic BM, it may be proper to initiate EGFR TKI therapy and defer upfront brain RT, particularly in those with active systemic disease. Prospective studies are needed to validate these clinical findings.

\section{Abbreviations}

BM: Brain metastasis; Cl: Confidence interval; CNS: Central nervous system; CT: Computed tomography; ECOG: Eastern Cooperative Oncology Group; EGFR: Epidermal Growth Factor Receptor; EGFR-TKIs: Epidermal growth factor receptor tyrosine kinase inhibitors; HR: Hazard ratio; MRI: Magnetic resonance imaging; NSCLC: Non-Small Cell Lung Cancer; OS: Overall survival;

PET: Positron emission tomography; PFS: Progression-free survival; PR: Partial response; RT: Radiotherapy; SD: Stable disease; TKI: Tyrosine kinase inhibitors; TNM: Tumor/node/metastasis; WBRT: Whole-brain radiation therapy.

\section{Competing interests}

The authors declare that they have no competing interests.

\section{Authors' contributions}

SRL and BQ made substantial contributions to conception and design. SRL and ZLC carried out acquisition of data. LKC, FW, YL, PQC and SLL participated in the design of the study and performed the statistical analysis. LZ and MZL have given final approval of the version to be published. $\mathrm{HL}$ drafted the manuscript and revised it critically for important intellectual content. All authors read and approved the final manuscript.

\section{Author details}

${ }^{1}$ State Key Laboratory of Oncology in South China, Collaborative Innovation Center for Cancer Medicine, Guangzhou 510060, China. ${ }^{2}$ Guangdong Esophogeal Cancer Research Institute, Guangzhou, China. ${ }^{3}$ Department of Radiation Oncology, Sun Yat-sen University Cancer Center, State Key Laboratory of Oncology in South China; Collaborative Innovation Center for Cancer Medicine, Guangzhou 510060, China. ${ }^{4}$ Department of Medical Oncology, Sun Yat-sen University Cancer Center, Guangzhou, China. ${ }^{5}$ Department of Molecular Diagnosis, Sun Yat-sen University Cancer Center, Guangzhou, China. ${ }^{6}$ Department of Radiology, Sun Yat-sen University Cancer Center, Guangzhou, China.

Received: 12 February 2015 Accepted: 14 May 2015 Published online: 27 May 2015

\section{References}

1. Langer CJ, Mehta MP. Current management of brain metastases, with a focus on systemic options. J Clin Oncol. 2005;23:6207-19.

2. Sundstrom JT, Minn H, Lertola KK, Nordman E. Prognosis of patients treated for intracranial metastases with whole brain irradiation. Ann Med. 1998:30:296-9.

3. Khuntia D, Brown P, Li J, Mehta MP. Whole-brain radiotherapy in the management of brain metastasis. J Clin Oncol. 2006;24:1295-304.

4. Paz-Ares L, Soulieres D, Melezinek I, Moecks J, Keil L, Mok T, et al. Clinical outcomes in non-small-cell lung cancer patients with EGFR mutations: pooled analysis. J Cell Mol Med. 2010;14:51-69.

5. Lynch TJ, Bell DW, Sordella R, Gurubhagavatula S, Okimoto RA, Brannigan BW, et al. Activating mutations in the epidermal growth factor receptor underlying responsiveness of non-small-cell lung cancer to gefitinib. N Engl J Med. 2004;305:2129-39.

6. Mok TS, Wu YL, Thongprasert S, Yang CH, Chu DT, Saijo N, et al. Gefitinib or carboplatin-paclitaxel in pulmonary adenocarcinoma. N Engl J Med. 2009;361:947-57. 
7. Li Z, Guo H. The retrospective analysis of the frequency of EGFR mutations and efficacy of gefitinib in NSCLC patients with brain metastases. J Clin Oncol. 2011;29:e18065

8. Wu C, Li YL, Wang ZM, Li Z, Zhang TX, Wei Z. Gefitinib as palliative therapy for lung adenocarcinoma metastatic to the brain. Lung Cancer. 2007;57:359-64.

9. Kim JE, Lee DH, Choi Y, Yoon DH, Kim SW, Suh C, et al. Epidermal growth factor receptor tyrosine kinase inhibitors as a first-line therapy for neversmokers with adenocarcinoma of the lung having asymptomatic synchronous brain metastasis. Lung Cancer. 2009:65:351-4.

10. Eichler AF, Kahle KT, Wang DL, Joshi VA, Willers H, Engelman JA, et al. EGFP mutation status and survival after diagnosis of brain metastasis in nonsmall cell lung cancer. Neuro Oncol. 2010;12:1193-9.

11. Gow CH, Chien CR, Chang YL, Chiu YH, Kuo SH, Shih JY, et al. Radiotherapy in lung adenocarcinoma with brain metastases: effects of activating epidermal growth factor receptor mutations on clinical response. Clin Cancer Res. 2008;14:162-8.

12. Cappuzzo F, Ardizzoni A, Soto-Parra H, Gridelli C, Maione P, Tiseo M, et al. Epidermal growth factor receptor targeted therapy by ZD 1839 (Iressa) in patients with brain metastases from non-small cell lung cancer (NSCLC). Lung Cancer. 2003;41:227-31.

13. Lee HL, Chung TS, Ting LL, Tsai JT, Chen SW, Chiou JF, et al. EGFR mutations are associated with favorable intracranial response and progression-free survival following brain irradiation in non-small cell lung cancer patients with brain metastases. Radiat Oncol. 2012;7:181.

14. Omuro AM, Kris MG, Miller VA, Franceschi E, Shah N, Milton DT, et al. High incidence of disease recurrence in the brain and leptomeninges in patients with nonsmall cell lung carcinoma after response to gefitinib. Cancer. 2005;103:2344-8

15. Wang F, Fu S, Shao Q, Zhou YB, Zhang X, Zhang X, et al. High EGFR copy number predicts benefits from tyrosine kinase inhibitor treatment for nonsmall cell lung cancer patients with wild-type EGFR. J Transl Med. 2013;11:90.

16. Patchell RA, Tibbs PA, Walsh JW, Dempsey RJ, Maruyama Y, Kryscio RJ, et al. A randomized trial of surgery in the treatment of single metastases to the brain. N Engl J Med. 1990;322:494-500.

17. Tsao MN, Lloyd N, Wong RK, Chow E, Rakovitch E, Laperriere N, et al. Whole brain radiotherapy for the treatment of newly diagnosed multiple brain metastases. Cochrane Database Syst Rev. 2012;4:CD003869.

18. Akhtar MS, Kousar F, Fatmi S, Jabeen K, Akhtar K. Quality of life and symptoms control in brain metastasis after palliative whole brain radiotherapy using two different protocols. J Coll Physicians Surg Pak. 2012;22:311-6.

19. Hendriks LE, Troost EG, Steward A, Bootsma GP, De Jaeger K, van den Borne $B E$, et al. Patient selection for whole brain radiotherapy (WBRT) in a large lung cancer cohort: Impact of a new Dutch guideline on brain metastases. Acta Oncol. 2014;53:945-51.

20. El Gantery MM, El Baky HM, El Hossieny HA, Mahmoud M, Youssef O. Management of brain metastases with stereotactic radiosurgery alone versus whole brain irradiation alone versus both. Radiat Oncol. 2014;9:116.

21. Soffietti R, Kocher M, Abacioglu UM, Villa S, Fauchon F, Baumert BG, et al. A European Organisation for Research and Treatment of Cancer phase III trial of adjuvant whole-brain radiotherapy versus observation in patients with one to three brain metastases from solid tumors after surgical resection or radiosurgery: quality-of-life results. J Clin Oncol. 2013;31:65-72.

22. Matsumoto S, Takahashi K, Iwakawa R, Matsuno Y, Nakanishi Y, Kohno T, et al. Frequent EGFR mutations in brain metastases of lung adenocarcinoma. Int J Cancer. 2006;119:1491-4.

23. Huang SF, Liu HP, Li LH, Ku YC, Fu YN, Tsai HY, et al. High frequency of epidermal growth factor receptor mutations with complex patterns in non-small cell lung cancers related to gefitinib responsiveness in Taiwan. Clin Cancer Res. 2004;10:8195-203.

24. Wu YL, Zhong WZ, Li LY, Zhang XT, Zhang L, Zhou CC, et al. Epidermal growth factor receptor mutations and their correlation with gefitinib therapy in patients with non-small cell lung cancer: a meta-analysis based on updated individual patient data from six medical centers in mainland China. J Thorac Oncol. 2007;2:430-9.

25. Heimberger AB, Learn CA, Archer GE, McLendon RE, Chewning TA, Tuck FL, et al. Brain tumors in mice are susceptible to blockade of epidermal growth factor receptor (EGFR) with the oral, specific, EGFR-tyrosine kinase inhibitor ZD1839 (iressa). Clin Cancer Res. 2002;8:3496-502.
26. Park SJ, Kim HT, Lee DH, McLendon RE, Chewning TA, Tuck FL, et al. Efficacy of epidermal growth factor receptor tyrosine kinase inhibitors for brain metastasis in non-small cell lung cancer patients harboring either exon 19 or 21 mutation. Lung Cancer. 2012;77:556-60.

27. Gerber NK, Yamada Y, Rimner A, Shi W, Riely GJ, Beal K, et al. Erlotinib versus radiation therapy for brain metastases in patients with EGFR-mutant lung adenocarcinoma. Int J Radiat Oncol Biol Phys. 2014;89:322-9.

28. Sekine A, Kato T, Hagiwara E, Shinohara T, Komagata T, Iwasawa T, et al. Metastatic brain tumors from non-small cell lung cancer with EGFR mutations: distinguishing influence of exon 19 deletion on radiographic features. Lung Cancer. 2012;77:64-9.

29. Kalikaki A, Koutsopoulos A, Trypaki M, Souglakos J, Stathopoulos E, Georgoulias V, et al. Comparison of EGFR and K-RAS gene status between primary tumours and corresponding metastases in NSCLC. Br J Cancer. 2008;99:923-9.

30. Gow CH, Chang YL, Hsu YC, Tsai MF, Wu CT, Yu CJ, et al. Comparison of epidermal growth factor receptor mutations between primary and corresponding metastatic tumors in tyrosine kinase inhibitor-naive non-small-cell lung cancer. Ann Oncol. 2009;20:696-702.

\section{Submit your next manuscript to BioMed Central and take full advantage of:}

- Convenient online submission

- Thorough peer review

- No space constraints or color figure charges

- Immediate publication on acceptance

- Inclusion in PubMed, CAS, Scopus and Google Scholar

- Research which is freely available for redistribution 